\title{
6G Green IoT Network: Joint Design of Intelligent Reflective Surface and Ambient Backscatter Communication
}

\author{
Qiang Liu $\mathbb{D},{ }^{1}$ Songlin Sun $\mathbb{D}^{1},{ }^{1}$ Heng Wang $\mathbb{D},{ }^{2}$ and Shaowei Zhang $\mathbb{D}^{2}$ \\ ${ }^{1}$ School of Information and Communication Engineering, Beijing University of Posts and Telecommunications, Beijing, China \\ ${ }^{2}$ China Telecom Research Institute, Beijing, China \\ Correspondence should be addressed to Heng Wang; wangh26@chinatelecom.cn
}

Received 25 March 2021; Revised 24 May 2021; Accepted 4 June 2021; Published 22 June 2021

Academic Editor: Shengjie Xu

Copyright (c) 2021 Qiang Liu et al. This is an open access article distributed under the Creative Commons Attribution License, which permits unrestricted use, distribution, and reproduction in any medium, provided the original work is properly cited.

\begin{abstract}
Ambient backscatter communication $(\mathrm{AmBC})$ is one of the candidate solutions for the $6 \mathrm{G}$ green internet of things (IoT) network. However, the uncontrollability of the radio frequency $(\mathrm{RF})$ environment is one of the main obstacles hindering the popularization of AmBC. The intelligent reflective surface (IRS) can improve the radio frequency environment by adjusting the phase and amplitude of the incident signal, which provides the possibility for the widespread deployment of AmBC. Currently, there is no discussion about the joint optimization of AmBC and IRS. In this paper, we introduce a novel IRS and AmBC joint design method. The purpose of this method is to jointly design the beamforming vector, the IRS phase shift, and the reflection coefficient of AmBC to minimize the AP's transmit power while ensuring the quality of service of the AmBC system and the primary communication system. Due to the nonconvexity of the problem, the time complexity of solving the problem through exhaustive search will be very high. Therefore, we propose a joint design method based on an iterative beamforming vector, IRS phase shift, and reflection coefficient to minimize the AP's transmit power. This method can effectively reduce the transmission power of the access point (AP), and the simulation results prove the effectiveness of the method.
\end{abstract}

\section{Introduction}

With the rapid development of mobile communication technology, the internet of things (IoT) has been greatly developed and popularized. In particular, $\mathrm{B} 5 \mathrm{G} / 6 \mathrm{G}$ further promotes the application range of IoT, such as smart home, smart manufacturing, and smart cities $[1,2]$. However, due to the increase in diversified requirements for the application scenarios of the IoT, diversified requirements are also put forward for the needs of the IoT devices. If the IoT device $[3,4]$ actively generates signals for wireless communication, it will consume a lot of energy, which will undoubtedly reduce the standby time of the device. Increasing the battery capacity will increase the standby time of the device, but this will undoubtedly increase the size and cost of the device. Especially for IoT devices such as wearable devices, they are very sensitive to device size and standby time. The battery capacity and size of IoT devices are the restrictive factors for their widespread popularity. Therefore, low-energy IoT device transmission solutions are an important research direction to realize the potential of the IoT [3-7].

Radio frequency (RF) energy harvesting technology can obtain energy from external radio frequency sources and is one of the important research directions of low-energy consumption IoT device transmission solutions. RF energy harvesting technology has been widely used in low-power IoT devices. Wireless IoT devices can use RF energy harvesting technology to collect energy to maintain their normal operations. In this way, the wireless device can run for a long time without any manual intervention, thereby reducing the operation and maintenance costs of the device. Therefore, RF energy harvesting is particularly suitable for powerconstrained wireless networks. There are three main types of RF energy harvesting schemes, including the synchronous wireless information and power transmission network (SWIPT), wireless power communication network (WPCN), and wireless power transmission (WPT) [8]. (1) The SWIPT scheme allows the transmitter to send information and 
energy at the same time, and the user can choose to decode the information or collect energy. (2) The WPCN scheme allows user equipment to collect energy from RF energy signals and then actively send data. (3) The WPT scheme allows the power transmitter to transmit energy to the user equipment. Although these solutions have their application value in wireless networks, there are still some limitations. First, these solutions require a dedicated RF source to send RF energy or information to users. Secondly, active RF data transmission requires a complicated circuit design and consumes a lot of power.

As a green communication technology, ambient backscatter communication (AmBC) can effectively solve the above-mentioned limitations of traditional radio frequency energy harvesting technology $[8,9]$. In the AmBC system, backscatter devices can communicate by using broadcast signals from RF sources such as cellular base stations, FM towers, and TV towers. In the AmBC system, the backscatter transmitter can modulate the data to the surrounding ambient signal and reflect it to the backscatter receiver. Therefore, AmBC does not need a dedicated frequency spectrum for data transmission. Therefore, $\mathrm{AmBC}$ has advantages that other communication methods do not have. First, AmBC does not require a dedicated spectrum for data transmission, which improves spectrum utilization. Secondly, since AmBC does not require a dedicated $\mathrm{RF}$ source, maintenance costs and deployment costs are reduced. These advantages can make AmBC widely used in many practical applications. AmBC has huge application potential in future low-energy scenarios, but it still faces many challenges. The quality of service (QoS) of AmBC is affected by factors such as the location of the RF, the type of RF, and the RF environment. Therefore, AmBC must be designed specifically for specific RF sources. In addition, to use ambient signals from licensed sources, the AmBC protocol must ensure that it does not interfere with the QoS of licensed users.

Intelligent reflective surfaces (IRSs) [10-12] can realize an intelligent and reconfigurable radio propagation environment for the B5G/6G wireless communication system [1320]. The IRS is a plane containing a large number of lowcost passive reflective elements, each of which can independently change the phase and/or amplitude of the incident signal. The IRS can improve the required channel conditions, thereby achieving a substantial increase in wireless communication capacity and reliability. Intelligent reflective surfaces (IRSs) also have various practical advantages in implementation. First, compared with traditional active antenna arrays, IRS can only passively reflect impact signals without generating radio frequency resonance. Second, IRS does not have any noise amplification and self-interference. Third, due to the simple structure of the IRS, it can be easily deployed in any desired location. Finally, IRS has good compatibility and compatibility and can be integrated into existing communication systems.

There are many studies on AmBC or IRS [3-11], but there are no articles on the joint optimization design of AmBC and IRS. For example, [9] evaluated the performance of the environmental backscattering system but did not consider the role of IRS. Reference [10] used the IRS to enhance the active communication system to achieve the goal of minimum transmission power. Reference [11] combined IRS beamforming and reflection design to enhance Bistatic Backscatter Networks. IRS is a means to optimize the performance of $\mathrm{AmBC}$, so it is necessary to study the joint optimization design of IRS and AmBC. Therefore, in this article, we have conducted a joint optimization design for IRS and AmBC to ensure the quality of service of active communication and $A m B C$ while minimizing the transmission power. The innovations of this paper are as follows:

(1) We considered an IRS-assisted spectrum sharing system, where AmBC rides on the primary communication system. The receivers in the two systems are the same receiver and can demodulate the signals of the two systems. We call this receiver a cooperative receiver (CR). Specifically, after the CR demodulates the signal of the primary communication system, the signal of the AmBC is then demodulated based on the demodulated signal

(2) Under the condition that both the main communication system and $\mathrm{AmBC}$ are constrained by the quality of service, we have studied the issue of the minimum transmit power of the access point (AP) based on IRS assistance. This problem is nonconvex, so convex optimization methods cannot be used directly to solve this problem. At the same time, to solve this problem through exhaustive search methods, the time complexity will be very high. Therefore, we propose an iterative optimization method to optimize the minimum transmit power of the AP. Through joint beamforming and IRS phase shift design, the proposed iterative optimization method can effectively reduce the minimum transmit power of the AP

The rest of this paper is organized as follows. Section 2 introduces system model and problem formulation. Section 3 presents the optimization algorithm based on an alternate iteration. Section 4 presents numerical results and Section 5 concludes the paper.

Notations: scalars are represented by italic letters, vectors are represented by bold lowercase letters, and matrices are represented by bold uppercase letters. $|x|$ represents the modulus of the complex number. $\|\mathbf{x}\|$ represents the Euclidean norm of the complex-valued vector $\mathbf{x}$. diag $(\mathbf{x})$ represents a diagonal matrix, and each diagonal item is a corresponding item in $\mathbf{x} . \operatorname{tr}(\mathbf{X})$ represents the trace of the square matrix $\mathbf{X}$. $\mathbf{X} \geqslant 0$ means $\mathbf{X}$ is a positive semidefinite matrix.

\section{System Model and Problem Formulation}

2.1. System Setup. The intelligent reflective surface- (IRS-) enhanced spectrum sharing system includes a primary communication system and a secondary communication system, as shown in Figure 1. The primary communication system is a MISO downlink communication system, which consists of a receiver and an access point (AP) with $M$ antennas. The secondary communication system is an AmBC system, which consists of a receiver and a backscatter device (BD). 


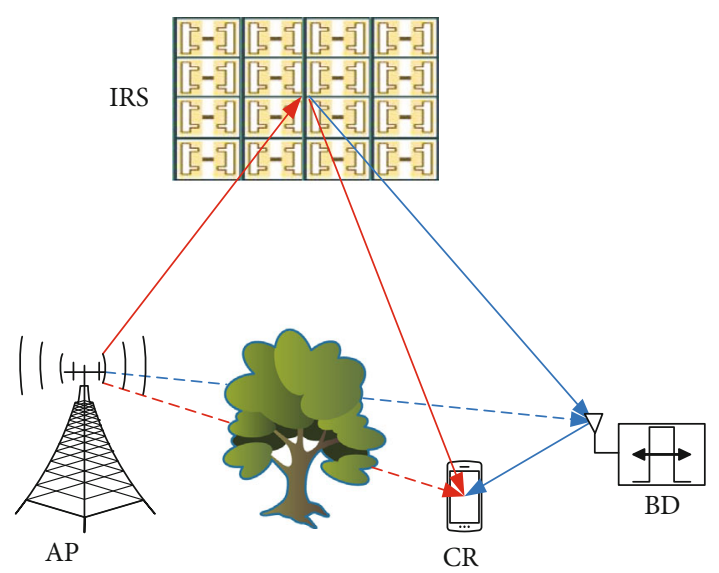

FIGURE 1: IRS-enhanced spectrum sharing system.

The backscatter device in the secondary system is a passive device, and its information transmission depends on the AP signal. We assume that the receiver in the primary communication system and the receiver in the secondary communication system are the same receiver. In other words, the receiver receives and demodulates the signal from the backscatter transmitter and the signal from the BS, simultaneously. For ease of expression, we denote the receiver as the cooperative receiver (CR). To improve QoS, an IRS with $N$ passive reflective elements is used to help this spectrum sharing system communicate. The IRS equipped with an intelligent controller can be based on the signal propagation environment, and each reflective element can dynamically adjust the amplitude and phase shift of the incident signal.

2.2. IRS Model. IRS is a very promising green communication technology, which can reconfigure the wireless propagation environment through software. The IRS can modify the wireless channel between the transmitter and the receiver through a highly controllable reflection unit. This paved the way for the realization of a controllable wireless environment. Since IRS has no RF link, it has the advantages of low cost and low-energy consumption. Because the beam of the IRS is controllable, there is no need for complex interference management between IRSs. Assuming that the IRS is a frequency-selective surface, it allows certain RF signals to pass, absorb, or reflect certain signals. That is to say, IRS can reflect RF signals in a specific frequency band but cannot reflect RF signals in other frequency bands.

The IRS consists of $N$ reflect elements, and each element $n \in\{1,2, \cdots, N\}$ can reflect the incident signal with a complex reflection coefficient. The complex reflection coefficient of the $n$th reflection element can be expressed as $\beta_{n} e^{j \theta_{n}}$, where $\beta_{n} \epsilon[0,1], \forall n \in\{1,2, \cdots, N\}$ is the amplitude gain and $\theta_{n} \epsilon[0,2 \pi), \forall n \in\{1,2, \cdots, N\}$ is the phase shift. Although in theory, the amplitude gain can be adjusted within the interval $[0,1]$. But adjusting amplitude gain and phase shift at the same time will greatly increase the complexity of the system. Therefore, without loss of generality, we take the upper bound of the interval $[0,1]$ as the amplitude gain of all reflection elements, i.e., $\beta_{n}=1, \forall n \in\{1,2, \cdots, N\}$. Then, the reflec- tion coefficient matrix can be written as $\Theta=\operatorname{diag}\left(e^{j \theta_{1}}, e^{j \theta_{2}}, \cdots e^{j \theta_{N}}\right)$.

2.3. Backscatter Model. Since both IRS and BD can reflect signals, the signal will be reflected multiple times between IRS and $\mathrm{BD}$, which greatly complicates the problem. We assume that the PT transmits a continuous wave signal with carrier frequency $f_{c}$ and bandwidth $B$ to communicate with the $\mathrm{PR}$. In order to avoid the above-mentioned problems, BD adopts the following modulation method. First, BD uses a method similar to FSK modulation to shift the signal frequency $f_{c}$ to frequency $f_{c}+\Delta f_{c}$ (only performs frequency shift; this process does not carry BD data) and then modulates the data that $\mathrm{BD}$ needs to send at frequency $f_{c}+\Delta f_{c}$, where $\Delta f_{c}$ represents the frequency shift of the carrier frequency after BD modulation. Assume that the IRS can only reflect the signals with a specific frequency and bandwidth. Based on the difference in channel conditions, we assume that the IRS allows the reflection of the RF signal with carrier frequency $f_{c}$ and bandwidth $B$ but cannot reflect the RF signal with carrier frequency $f_{c}+\Delta f_{c}$ and bandwidth $B$. Therefore, to ensure that the signal sent by the PT and the signal reflected by the $\mathrm{BD}$ do not overlap in frequency, $\Delta f$ needs to meet the constraint condition $\Delta f \geq B$. Although the above process occupies additional spectrum resources, it can effectively solve the problem of multiple reflections between IRS and BD.

In the process of ambient backscatter communication, we need to consider the power consumption constraints of the $\mathrm{BD}$ circuit; that is, the ambient signal energy received by the $\mathrm{BD}$ must meet the circuit power consumption constraints to activate the $\mathrm{BD}$ circuit for backscatter communication. Assuming that the minimum received signal power to maintain the normal operation of the $\mathrm{BD}$ circuit is $P_{\min }$. When the $\mathrm{BD}$ is semipassive, the $\mathrm{BD}$ needs other power sources to supply power. In this case, all received signals are used for reflection communication. When $\mathrm{BD}$ is passive, the energy of the input signal must be greater than $P_{\min }$. In this case, part of the received signal is used to power the $\mathrm{BD}$ circuit, and the other part is used for scatter communication. Since the passive BD has no power supply battery, its volume and cost have advantages compared with the semipassive BD. Therefore, in the following analysis, we mainly consider passive BD.

2.4. Transmission Model. We assume that the channel is flat fading and does not change during the coherence time. Then, we denote the channels of AP-IRS, BS-CR, AP-BD, AP-CR, IRS-CR, IRS-BD, and BD-CR as $\mathbf{H}_{a i} \in \mathbb{C}^{N \times M}, h_{a c}^{H} \in \mathbb{C}^{1 \times M}, h_{a b}^{H}$ $\in \mathbb{C}^{1 \times M}, h_{c i}^{H} \in \mathbb{C}^{1 \times N}, h_{b i}^{H} \in \mathbb{C}^{1 \times N}$, and $h_{b c} \in \mathbb{C}^{1 \times 1}$. We assume that $\mathrm{CR}$ is assigned a linear beamforming vector, which can be denoted as $w \in \mathbb{C}^{M \times 1}$. Then, the signal transmitted by $\mathrm{AP}$ is given as follows:

$$
x_{a p}=w s
$$

where $s$ is the signal that the main communication system needs to send and $E\left(|s|^{2}\right)=1$. In this paper, we assume that IRS allows reflecting the RF signal with carrier frequency $f_{c}$ 
but cannot reflect the RF signal with carrier frequency $f_{c}+$ $\Delta f_{c}$. Then, the signal received by the DB is mainly composed of two parts: one part is from AP and the other part is reflected by IRS. The signal received by $\mathrm{BD}$ can be expressed as

$$
y_{b}=\left(\mathbf{h}_{b i}^{H} \Theta \mathbf{H}_{a i}+h_{a b}^{H}\right) w s .
$$

Since no signal processing is performed in the $\mathrm{BD}$, there is no noise term at (2), which is consistent with the backscatter literature. Since BD is a passive device, it needs to collect energy to power its circuit operation. Therefore, the signal received by the $\mathrm{BD}$ will be divided into two parts, which are used for circuit operation and signal reflection. Denote the reflection efficiency as $\alpha$, then the $\alpha$ needs to satisfy the following constraint:

$$
0<\alpha \ll 1
$$

Let $c$ denote the signal of $\mathrm{BD}$, then the signal reflected by $\mathrm{BD}$ is given by

$$
x_{b}=\sqrt{\alpha} y_{b} c \mathrm{e}^{\mathrm{j} 2 \pi \Delta \mathrm{ft}}
$$

The remaining part is used to support the normal operation of the $\mathrm{BD}$ circuit. The power of the signal input for energy harvesting can be expressed as

$$
P_{b}=(1-\alpha) \eta\left\|y_{b}\right\|^{2}
$$

where $\eta$ denotes the energy conversion efficiency of BD. Assume that the minimum power required to support the operation of the $\mathrm{BD}$ circuit is $P_{\min }$, then the following constraints should be satisfied:

$$
(1-\alpha) \eta\left\|y_{b}\right\|^{2} \gg P_{\min }
$$

Denote the received signal of $\mathrm{CR}$ as $y_{c}(n)$, which is mainly composed of the signal from AP, IRS, and BD. Then, $y_{c}(n)$ is given by

$$
y_{c}=\left(h_{c i}^{H} \Theta \mathbf{H}_{a i}+h_{a c}^{H}\right) w s(t)+h_{b c} x_{b}+n,
$$

where $n \in C N\left(0, \sigma^{2}\right)$ denotes the Gaussian noise. Then, the received signal plus noise ratio (SNR) of demodulated $s(n)$ at the $\mathrm{CR}$ is given by

$$
\gamma_{s}=\frac{\left|\left(\mathbf{h}_{c i}^{H} \Theta \mathbf{H}_{a i}+h_{a c}^{H}\right) w\right|^{2}}{\sigma^{2}} .
$$

We assume that the primary communication system has a minimum SINR requirement and denote it as $\gamma_{t h}^{p}$. Then, the QoS constraints of the primary communication system are given by

$$
\gamma_{s} \gg \gamma_{t h}^{p}
$$

After the CR successfully demodulates $s(n)$, the CR can decode the received signal $c(n)$ by performing successful interference cancellation (SIC). Then, the instantaneous received SNR of demodulated $c(n)$ at the $\mathrm{CR}$ is given by

$$
\gamma_{c}=\frac{\alpha\left|\left(\mathbf{h}_{b i}^{H} \Theta \mathbf{H}_{a i}+h_{a b}^{H}\right) h_{b c} w\right|^{2}}{\sigma^{2}} .
$$

We assume that $\mathrm{AmBC}$ has the minimum SNR requirement $\gamma_{t h}^{a}$. To ensure the QoS of AmBC, the following conditions must be met:

$$
\gamma_{c} \gg \gamma_{t h}^{a}
$$

2.5. Problem Formulation. We study the issue of minimum transmit power under the condition that CR and BD meet their SNR requirements. Therefore, we need to jointly optimize the beamforming vector of AP, the phase shift of IRS, and the backscatter coefficient of $\mathrm{BD}$ to minimize transmit power of AP. Then, the corresponding optimization problem can be written as

$$
\begin{gathered}
(\mathrm{P} 1): \max _{\Theta, \alpha . w}\|w\|^{2}, \\
\text { s.t. }(1-\alpha) \eta\left\|y_{b}\right\|^{2} \gg P_{\min }, \\
\frac{\left|\left(h_{c i}^{H} \Theta \mathbf{H}_{a i}+h_{a c}^{H}\right) w\right|^{2}}{\sigma^{2}} \gg \gamma_{t h}^{p}, \\
\frac{\alpha\left|\left(h_{b i}^{H} \Theta \mathbf{H}_{a i}+h_{a b}^{H}\right) h_{b c} w\right|^{2}}{\sigma^{2}} \gg \gamma_{t h}^{a}, \\
0 \ll \alpha \ll 1, \\
0 \leq \theta_{n} \ll 2 \pi, \quad \forall n=1,2, \cdots, N .
\end{gathered}
$$

Obviously, (P1) is a nonconvex problem. There is no optimal solution to this problem. Next, we will analyze and simplify this problem so that it can be solved effectively.

\section{Optimization Algorithm Based on Alternate Iteration}

It can be seen that the problem (P1) is affected by multiple variables, which makes the problem difficult to solve. We use alternating optimization to solve problem (P1), which iteratively optimizes one variable while holding the others constant. In this section, we will introduce in detail how to solve problem (P1).

3.1. Transmit Beamforming Vector Optimization. When the phase of the IRS $\Theta$ and the reflection efficiency of the BD $\alpha$ are fixed, the vectors $\mathbf{h}_{c i}^{H} \Theta \mathbf{H}_{a i}+h_{a c}^{H}, \mathbf{h}_{b i}^{H} \Theta \mathbf{H}_{a i}+h_{a b}^{H}$, and $\left(\mathbf{h}_{b i}^{H}\right.$ $\left.\Theta \mathbf{H}_{a i}+h_{a b}^{H}\right) h_{b c}$ are fixed. Define $g_{a c}=\mathbf{h}_{c i}^{H} \Theta \mathbf{H}_{a i}+h_{a c}^{H}, g_{a b}=$ $\mathbf{h}_{b i}^{H} \Theta \mathbf{H}_{a i}+h_{a b}^{H}$, and $g_{b c}=\left(\mathbf{h}_{b i}^{H} \Theta \mathbf{H}_{a i}+h_{a b}^{H}\right) h_{b c}$, then problem (P1) can be expressed as 


$$
\begin{gathered}
(\mathrm{P} 2): \min _{w}\|w\|^{2}, \\
\text { s.t. }(1-\alpha) \eta\left|g_{a b} w\right|^{2} \gg P_{\min }, \\
\frac{\left|g_{a c} w\right|^{2}}{\sigma^{2}} \gg \gamma_{t h}^{p}, \\
\frac{\alpha\left|g_{b c} w\right|^{2}}{\sigma^{2}} \gg \gamma_{t h}^{a} .
\end{gathered}
$$

By observing (P2), we noticed that $\|w\|^{2}=\operatorname{tr}\left(w w^{H}\right)$, $\left|g_{a c} w\right|^{2}=\operatorname{tr}\left(w w^{H} g_{a c}^{H} g_{a c}\right)$, and $\left|g_{a b} w\right|^{2}=\operatorname{tr}\left(w w^{H} g_{a b}^{H} g_{a b}\right)$. Define $\mathrm{X}=w w^{H}, G_{a c}=g_{a c}^{H} g_{a c}, G_{a b}=g_{a b}^{H} g_{a b}$, and $G_{b c}=g_{b c}^{H}$ $g_{b c}$. Then, problem (P2) can be equivalently written as

$$
\begin{aligned}
& \text { (P3): } \min _{X} \operatorname{tr}(X) \text {, } \\
& \text { s.t. } \operatorname{tr}\left(X G_{a b}\right)(1-\alpha) \eta \gg P_{\min } \text {, } \\
& \operatorname{tr}\left(X G_{a c}\right) \gg \gamma_{t h}^{p} \sigma^{2}, \\
& \operatorname{tr}\left(X G_{b c}\right) \gg \gamma_{t h}^{a} \sigma^{2}, \\
& X \geqslant 0 \text {, } \\
& \operatorname{rank}(X)=1,
\end{aligned}
$$

where problem (14a) is linear in $X$, then constraints (14b)-(14d) are linear inequalities in $X . X \geqslant 0$ means that the matrix $X$ is a symmetric positive semidefinite matrix, and the set of symmetric positive semidefinite matrices is convex. Note that the rank constraint in (14d) is the only nonconvex constraint. Therefore, we can use the SDR method to relax this constraint. Then, problem (P3) can be rewritten as

$$
\begin{gathered}
(\mathrm{P} 4): \min _{X} \operatorname{tr}(\mathrm{X}), \\
\text { s.t.tr }\left(\mathrm{X} G_{a b}\right)(1-\alpha) \eta \gg P_{\min }, \\
\operatorname{tr}\left(X G_{a c}\right) \gg \gamma_{t h}^{p} \sigma^{2}, \\
\operatorname{tr}\left(X G_{b c}\right) \gg \gamma_{t h}^{a} \sigma^{2}, \\
X \geqslant 0 .
\end{gathered}
$$

Obviously, problem (P4) is a standard convex semidefinite program (SDP), which can be optimized by a convex optimization solver such as CVX. Generally, the rank of the solution of problem (P4) is generally not equal to 1 , which means that the optimal value of (P4) is the lower bound to satisfy (P3). Therefore, the solution of problem (P4) needs to be further processed to satisfy the constraint of problem (P3). First, we eigenvalue decomposition of $X$ as $X=U \Sigma$ $U^{H}$, where $U$ is a unitary matrix and $\Sigma$ is a diagonal matrix. Then, a suboptimal solution of problem (P3) can be expressed as $w=U \Sigma^{1 / 2} e$, where $e$ is uniformly distributed on the unit sphere. $w$ may not satisfy the constraints of (15b)-(15d). However, all constraints can be satisfied by simply scaling $w$ to find a feasible weight vector.
3.2. IRS Phase Shift Optimization. Since the objective function (14a) in the problem (P1) depends only on $w$, the optimization of $\Theta$ can take the form of a feasibility problem. When $w$ and $\alpha$ are given, problem (P1) can be expressed as

(P5): find $\Theta$,

$$
\begin{gathered}
\text { s.t. }(1-\alpha) \eta\left|g_{a b} w\right|^{2} \gg P_{\min }, \\
\frac{\left|g_{a c} w\right|^{2}}{\sigma^{2}} \gg \gamma_{t h}^{p}, \\
\frac{\alpha\left|g_{b c} w\right|^{2}}{\sigma^{2}} \gg \gamma_{t h}^{a},
\end{gathered}
$$

$$
0 \leq \theta_{n} \ll 2 \pi, \quad \forall n=1,2, \cdots, N .
$$

Let $\mathbf{v}=\left[v_{1}, v_{2}, \cdots, v_{N}\right]$, where $v_{n}=e^{j \theta_{\mathrm{n}}}, \forall n=1,2, \cdots, N$. Then, the constraints in (16f) are equivalent to $\left|v_{n}\right|=1, \forall n$ $=1,2, \cdots, N$. In problem (P5), the variables related to $\Theta$ are $g_{a b}, g_{a c}$, and $g_{b c}$, so we need to change the forms of $g_{a b}, g_{q c}$ , and $g_{b c}$ to get the ideal expressions. By observing $g_{a b}=\mathbf{h}_{b i}^{H}$ $\Theta \mathbf{H}_{a i}+h_{a b}^{H}$ and $g_{a c}=\mathbf{h}_{c i}^{H} \Theta \mathbf{H}_{a i}+h_{a c}^{H}, g_{a b}$ and $g_{a c}$ can be rewritten as $g_{a b}=\overline{\mathbf{h}}_{b i}^{H} \bar{\Theta} \overline{\mathbf{H}}_{a i}$ and $g_{a c}=\overline{\mathbf{h}}_{c i}^{H} \bar{\Theta} \overline{\mathbf{H}}_{a i}$, respectively, where $\overline{\mathbf{h}}_{b i}^{H}=\left[\mathbf{h}_{b i}^{H} 1\right], \overline{\mathbf{h}}_{c i}^{H}=\left[\mathbf{h}_{c i}^{H} 1\right], \bar{\Theta}=\operatorname{diag}\left(e^{j \theta_{1}}, e^{j \theta_{2}}, \cdots e^{j \theta_{N}}, 1\right)$, and $\overline{\mathbf{H}}_{a i}=\left[\mathbf{H}_{a i} h_{a b}^{H}\right]$. Similarly, $g_{b c}=\left(\mathbf{h}_{b i}^{H} \Theta \mathbf{H}_{a i}+h_{a b}^{H}\right) h_{b c}$ can be rewritten as $g_{b c}=\overline{\mathbf{h}}_{c i}^{H} \bar{\Theta} \overline{\mathbf{H}}_{a i} h_{b c}$. Substituting $g_{a b}=\overline{\mathbf{h}}_{b i}^{H} \bar{\Theta} \overline{\mathbf{H}}_{a i}$ into (16b), we can get

$$
(1-\alpha) \eta\left|\overline{\mathbf{h}}_{b i}^{H} \bar{\Theta} \overline{\mathbf{H}}_{a i} w\right|^{2} \gg P_{\min }
$$

Let $\Phi_{a b}=\operatorname{diag}\left(\overline{\mathbf{h}}_{b i}^{H}\right) \overline{\mathbf{H}}_{a i} w$ and $\overline{\mathbf{v}}=[\mathbf{v} 1]$, then $\left|\overline{\mathbf{h}}_{b i}^{H} \bar{\Theta} \overline{\mathbf{H}}_{a i} w\right|^{2}$ in (17) can expressed as $\left|\overline{\mathbf{h}}_{b i}^{H} \bar{\Theta} \overline{\mathbf{H}}_{a i} w\right|^{2}=\left|\overline{\mathbf{v}} \Phi_{a b}\right|^{2}$. Based on the following, the fact that $\left|\overline{\mathbf{v}} \Phi_{a b}\right|^{2}=\operatorname{tr}\left(\overline{\mathbf{v}} \Phi_{a b} \Phi_{a b}^{H} \overline{\mathbf{v}}^{H}\right)=\operatorname{tr}\left(\overline{\mathbf{v}}^{H} \overline{\mathbf{v}} \Phi_{a b} \Phi_{a b}^{H}\right)$, we have $\boldsymbol{V}$ $=\overline{\mathbf{v}}^{H} \overline{\mathbf{v}}$ and $\boldsymbol{R}_{a b}=\Phi_{a b} \Phi_{a b}^{H}$.Then, (17) can be rewritten as

$$
\operatorname{tr}\left(\mathbf{V R}_{a b}\right)(1-\alpha) \eta \gg P_{\min }
$$

Substituting $g_{a c}=\overline{\mathbf{h}}_{c i}^{H} \bar{\Theta} \overline{\mathbf{H}}_{a i}$ into (18), we can get

$$
\left|\overline{\mathbf{h}}_{c i}^{H} \bar{\Theta} \overline{\mathbf{H}}_{a i} w\right|^{2} \gg \gamma_{t h}^{p} \sigma^{2}
$$

Let $\Phi_{a c}=\operatorname{diag}\left(\overline{\mathbf{h}}_{c i}^{H}\right) \overline{\mathbf{H}}_{a i} w$ and $\Phi_{b c}=\Phi_{a b} h_{b c}$, then $\left|\overline{\mathbf{h}}_{c i}^{H} \bar{\Theta} \overline{\mathbf{H}}_{a i} w\right|^{2}$ in (19) can be expressed as $\left|\overline{\mathbf{h}}_{c i}^{H} \bar{\Theta} \overline{\mathbf{H}}_{a i} w\right|^{2}=\left|\overline{\mathbf{v}} \Phi_{a c}\right|^{2}$. Based on the following the fact that $\left|\overline{\mathbf{v}} \Phi_{a b}\right|^{2}=\operatorname{tr}\left(\overline{\mathbf{v}}^{H} \overline{\mathbf{v}} \Phi_{a c} \Phi_{a c}^{H}\right)$, we can have $\boldsymbol{R}_{a c}=\Phi_{a c} \Phi_{a c}^{H}$. Then, (19) can be rewritten as

$$
\operatorname{tr}\left(\mathbf{V R}_{a c}\right) \gg \gamma_{t h}^{p} \sigma^{2}
$$


According to $\left|\overline{\mathbf{v}} \Phi_{b c}\right|^{2}=\operatorname{tr}\left(\overline{\mathbf{v}}^{H} \overline{\mathbf{v}} \Phi_{b c} \Phi_{b c}^{H}\right)$ and $\quad \boldsymbol{V}=\overline{\mathbf{v}}^{H} \overline{\mathbf{v}}$ (16d) can be rewritten as

$$
\operatorname{tr}\left(\mathbf{V R}_{a b}\right)\left|h_{b c}\right|^{2} \alpha \gg \gamma_{t h}^{a} \sigma^{2}
$$

Then, problem (P5) can be expressed as

(P6): find V,

$$
\begin{gathered}
\text { s.t.tr }\left(\mathbf{V R}_{a b}\right)(1-\alpha) \eta \gg P_{\min }, \\
\operatorname{tr}\left(\mathbf{V}\left(\mathbf{R}_{a c}\right) \gg \gamma_{t h}^{p} \sigma^{2},\right. \\
\operatorname{tr}\left(\mathbf{V R}_{a b}\right)\left|h_{b c}\right|^{2} \alpha \gg \gamma_{t h}^{a} \sigma^{2}, \\
\mathbf{V} \geqslant 0, \\
\left|v_{n}\right|=1, \quad \forall n=1,2, \cdots, N, \\
\operatorname{rank}(\mathbf{V})=1 .
\end{gathered}
$$

To obtain an explicit solution of feasible $\mathbf{V}$, we can convert problem (P6) into an optimization problem based on constraints (22b)-(22f). Then, we introduce the slack variable $\mu$ to represent the difference between the achievable circuit constraint value and its requirement, then the optimization problem is as follows:

$$
\begin{gathered}
(\mathrm{P} 7): \max _{\mathbf{V}} \mu, \\
\text { s.t. } \operatorname{tr}\left(\mathbf{V} \mathbf{R}_{a b}\right)(1-\alpha) \eta \gg P_{\min }+\mu, \\
\operatorname{tr}\left(\mathbf{V R}_{a c}\right) \gg \gamma_{t h}^{p} \sigma^{2}, \\
\operatorname{tr}\left(\mathbf{V R}_{a b}\right)\left|h_{b c}\right|^{2} \alpha \gg \gamma_{t h}^{a} \sigma^{2}, \\
\mathbf{V} \geqslant 0, \\
\left|v_{n}\right|=1, \quad \forall n=1,2, \cdots, N, \\
\operatorname{rank}(\mathbf{V})=1 .
\end{gathered}
$$

By relaxing the rank constraint, i.e., $\operatorname{rank}(\mathbf{V})=1$, the problem can be transformed into a convex optimization problem, which can be easily solved using CVX. Then, we perform Gaussian randomization on the solution obtained by solving the CVX to obtain a rank-one solution. The process of Gaussian randomization is as follows. First, perform eigenvalue decomposition on $\mathbf{V}$ to get $\mathbf{V}=U \Sigma U^{H}$, where $U$ $=\left[e_{1}, e_{2}, \cdots, e_{N+1}\right]$ is a unitary matrix and $\Sigma=\operatorname{diag}\left(\lambda_{1}, \lambda_{1}\right.$, $\left.\cdots, \lambda_{N+1}\right)$ is a diagonal matrix. Then, let $\overline{\mathbf{v}}=e^{\operatorname{jarg}\left(U \Sigma^{1 / 2} r\right)}$, where $r \in C N(0,1)$. For the independently generated Gaussian random vector $r$, the target value $\overline{\mathbf{v}}$ is approximately the maximum one of problem (P7) among all $r$. Then, we can get the IRS phase shift $\mathbf{v}=e^{j[\overline{\mathbf{v}} / \overline{\mathbf{v}}[N+1]](1: N)}$, where $[\overline{\mathbf{v}} / \overline{\mathbf{v}}[N+1]](1$ $: N)$ represents a vector containing the first $N$ elements of $\overline{\mathbf{v}}$ $/ \overline{\mathbf{v}}[N+1]$.

3.3. Reflection Coefficient Optimization. Next, we will discuss the optimization method of reflection coefficient. The feasibility problem can be written as
(P8): find $\alpha$,

$$
\begin{gathered}
\text { s.t. }(1-\alpha) \eta\left|g_{a b} w\right|^{2} \gg P_{\min }, \\
\frac{\left|g_{a c} w\right|^{2}}{\alpha\left|g_{b c}\right|^{2}+\sigma^{2}} \gg \gamma_{t h}^{p}, \\
\frac{\alpha\left|g_{b c} w\right|^{2}}{\sigma^{2}} \gg \gamma_{t h}^{a}, \\
0 \ll \alpha \ll 1 .
\end{gathered}
$$

Based on (24b)-(24e), we can get the value range of $\alpha$. The value range of $\alpha$ can be expressed as follows:

$$
\frac{\sigma^{2} \gamma_{t h}^{a}}{\left|g_{b c} w\right|^{2}} \ll \alpha \ll \min \left(1-\frac{P_{\min }}{\eta\left|g_{a b} w\right|^{2}}, \frac{\left|g_{a c} w\right|^{2}}{\gamma_{t h}^{p}\left|g_{b c}\right|^{2}}-\frac{\sigma^{2}}{\left|g_{b c}\right|^{2}}\right)
$$

Obviously, the value of $\alpha$ is determined by $w$ and $\Theta$. Therefore, if the optimal values of $w$ and $\Theta$ cannot be determined, we cannot obtain the optimal value of $\alpha$ in a limited time.

However, by observing Algorithms 1 and 2, we can draw the following conclusions: for given $\alpha$ and $\Theta$, we can find the optimal $w$ of problem (P2) according to Algorithm 1; for given $\alpha$ and $w$, we can find the optimal $\Theta$ of problem (P5) according to Algorithm 2. Therefore, for a given $\alpha$, we can alternately use Algorithms 1 and 2 to solve the local optimal solution of problem (P1). To facilitate practical implementation, we consider that the backscattering coefficient can only adopt a limited number of discrete values. Let $L$ indicate the number of backscattering coefficient levels. For simplicity, we assume that such discrete backscattering coefficients are obtained by uniformly quantizing the interval $(0,1]$. Thus, the set of discrete backscattering coefficients is given by

$$
\mathscr{F}=\{\Delta \alpha, 2 \Delta \alpha, \cdots, 1\},
$$

where $\Delta \alpha=1 / L$. Let $\alpha_{l}=l \Delta \alpha, l=1,2,3, . ., L$. Then, we can solve the local optimal values $\Theta_{l}$ and $w_{l}$ for each $\alpha_{l}$ of problem $(\mathrm{P} 1)$. Let $W=\left[\left\|w_{1}\right\|^{2},\left\|w_{2}\right\|^{2}, \cdots,\left\|w_{L}\right\|^{2}\right]$. Then, the optimal solution of problem (P1) can be given by

$$
\left(w^{*}, \Theta^{*}, \alpha^{*}\right)=\arg \min (W) .
$$

Then, take the $\Theta_{l}, w_{l}$, and $\alpha_{i}$ that minimize $\left\|w_{l}\right\|^{2}$ as the solution to problem (P1). Algorithm 3 gives a detailed description of the alternate optimization algorithm, where $\varepsilon$ is a threshold for the increment of the objective value until convergence.

\section{Simulation Results}

In this section, we will evaluate the performance of the algorithm. To effectively evaluate the proposed algorithm, we consider the settings shown in Figure 2. The default locations 
1:Initialize: random IRS phase shifts $\Theta$; random backscatter coefficients $\alpha$; 2:Optimize problem (P4) by CVX and get $X$.

3:Get $U$ and $\Sigma$, where $X=U \Sigma U^{H}$

$4 \mathrm{Get} w=U \Sigma^{1 / 2} e$, where $e$ is uniformly distributed on the unit sphere.

5:Scaling $w$ so as to satisfy constraints (14b)-(14d).

Algorithm 1: Transmit beamforming vector optimization.

1:Initialize: backscatter coefficients $\alpha$ generated in Algorithm 1, transmit beamforming vector $w$ generated in Algorithm 1; number of Gaussian randomization $G$

2:Relax the constraint (23d), then optimize problem (P7) by CVX and get $\mathbf{V}$.

3:Perform eigenvalue decomposition $\mathbf{V}=U \Sigma U^{H}$

4:for $i=1$ to $G$ do

5:Get a rank-one solution of (P7): $\overline{\mathbf{v}}_{\mathbf{i}}=e^{\mathrm{jarg}\left(U \Sigma^{1 / 2} r\right)}$ end for

6:Obtain the target value of problem (P7), where $\overline{\mathbf{v}}=\max \mu\left(\overline{\mathbf{v}}_{\mathbf{i}}\right)$

7:Obtain the target value of problem (P5), where $\mathbf{v}=e^{\left.j \int \overline{\mathbf{v}} / \overline{\mathbf{v}}[N+1]\right](1: N)}$

Algorithm 2: IRS phase shift optimization.

1:Initialize: number of backscattering coefficient levels $L$, number of Gaussian randomization $G$, and threshold $\varepsilon$.

2:forl $=1$ to $L$ do

3:random IRS phase shifts $\Theta_{i}$

4:While the change of the objective function (12a) is higher than the threshold $\varepsilon$ do

5:Optimize problem (P4) by CVX and get $X$.

6:Get $U$ and $\Sigma$, where $X=U \Sigma U^{H}$

7:Get $w_{l}=U \Sigma^{1 / 2} e$, where $e$ is uniformly distributed. On the unit sphere.

8:Scaling $w_{l}$ so as to satisfy constraints (14b)-(14d).

9:Relax the constraint (23d), then optimize problem (P7) by CVX and get V.

10:Perform eigenvalue decomposition $\mathbf{V}=U \Sigma U^{H}$

11 :for $i=1$ to $G$ do

12:Get a rank-one solution of (P7), $\overline{\mathbf{v}}_{\mathbf{i}}=e^{\mathrm{jarg}\left(U \Sigma^{1 / 2} r\right)}$.

end for

13:Obtain the target value of problem (P7), where $\overline{\mathbf{v}}_{l}=\arg \max \mu\left(\overline{\mathbf{v}}_{\mathbf{i}}\right)$.

14: Obtain the target value of problem (P5), where $\mathbf{v}_{\mathbf{l}}=e^{j\left[\overline{\mathbf{v}}_{l} / \mathbf{v}_{\mathbf{l}}[N+1]\right](1: N)}$.

15:Set $\Theta_{l}=\operatorname{diag}\left(\mathbf{v}_{\mathbf{l}}\right)$ for the next iteration.

16: end while

17: $\left(w^{*}, \Theta^{*}, \alpha^{*}\right)=\arg \min \left(\left\|w_{l}\right\|^{2}\right)$.

18:end for

19:Return: optimized beamforming vector $w^{*}$, optimized phase shift vector $\Theta^{*}$, optimized reflection coefficient $\alpha^{*}$.

Algorithm 3: The alternate optimization algorithm.

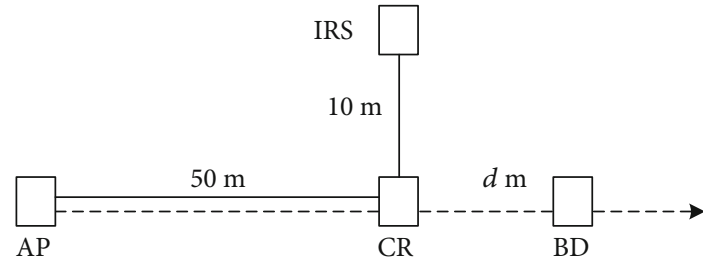

FIGURE 2: Simulation setup of the IRS-aided IRS spectrum sharing system. of the AP, IRS, CR, and BD are $(0,0),(50,10),(50,0)$, and $(d, 0)$, with all coordinates in meters hereafter.

The default number of IRS elements is $N=100$, while the AP has 8 antennas. We assume that all channels are independent Rayleigh fading, and the path loss index is set to 2.2 and the reference distance is $1 \mathrm{~m}$. For all channels, the path loss at 1 meter $(\mathrm{m})$ is set to $30 \mathrm{~dB}$. For ease of analysis, we assume that each channel coefficient is uniformly randomly generated from $[0,2 \pi)$. Since there is occlusion between AP and $\mathrm{CR}(\mathrm{BD})$, we assume that the penetration loss is $10 \mathrm{~dB}$. At the same time, we assume that the antenna gain of $\mathrm{AP}, \mathrm{BD}$, and $\mathrm{CR}$ are all $0 \mathrm{dBi}$ and the antenna gain of each reflective 


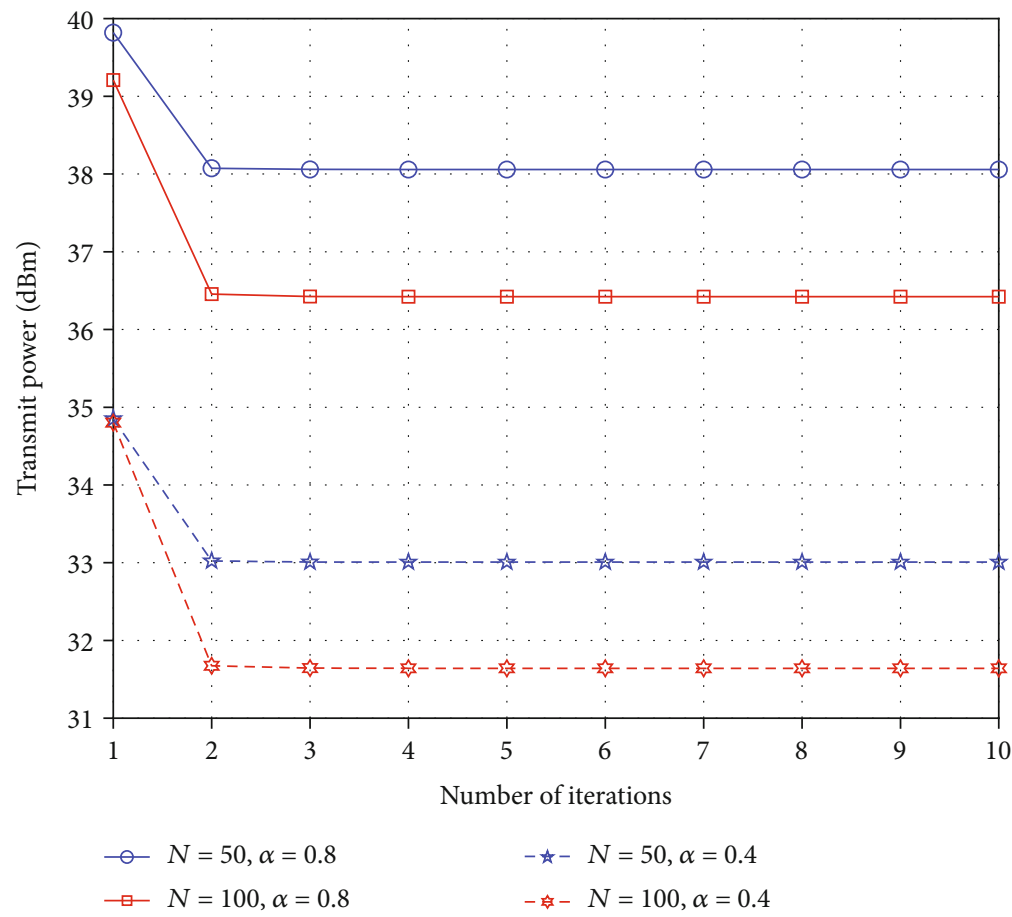

FIGURE 3: Convergence of the proposed distributed algorithm.

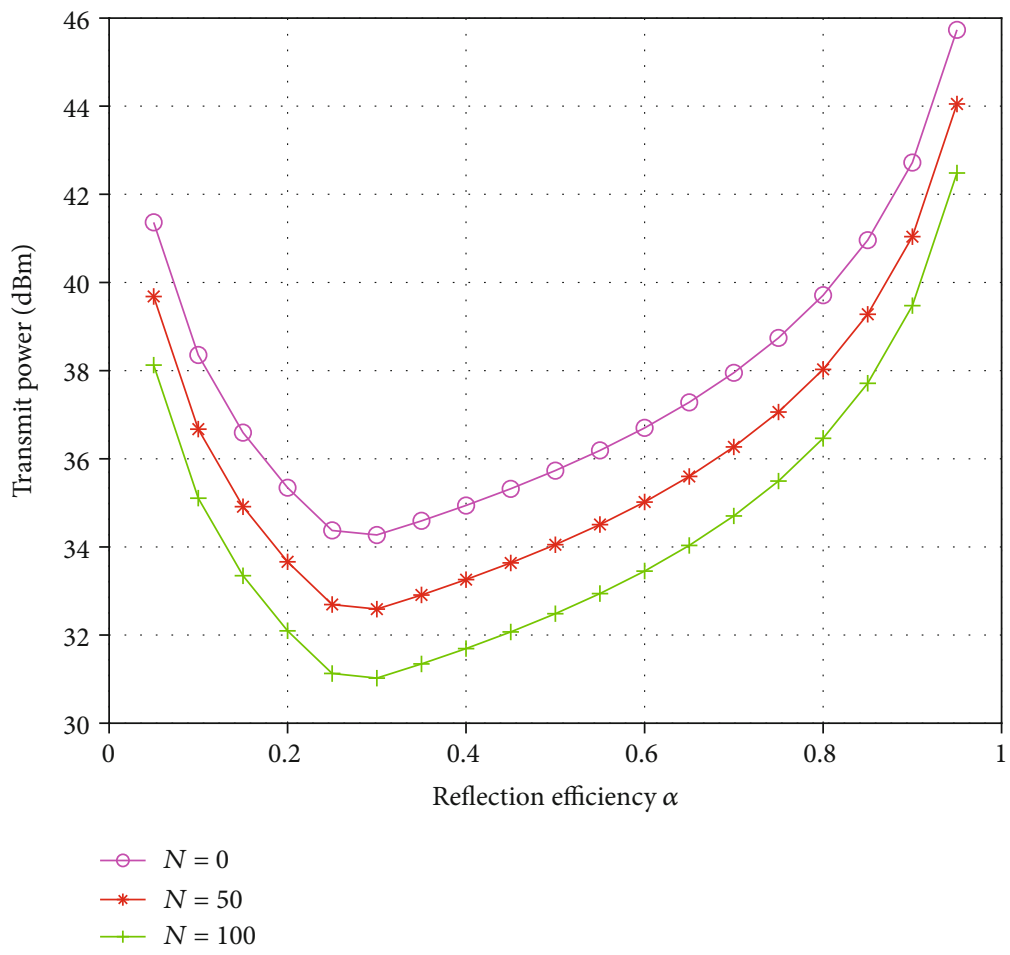

FIgure 4: The influence of the number of reflection units.

element of the IRS is $5 \mathrm{dBi}$. The noise power is set to $-90 \mathrm{dBm}$. The threshold $\varepsilon$ is set to 0.01 . We set the minimum SNR required to demodulate the primary signal and the $\mathrm{BD}$ signal to $20 \mathrm{~dB}$ and $13 \mathrm{~dB}$, respectively.

First, we verified the convergence of the algorithm. When verifying the convergence of the algorithm, we do not con- sider the impact of the threshold on the algorithm but only consider the impact of the number of iterations on the algorithm. To qualitatively analyze the convergence of the algorithm, we locate $\mathrm{BD}$ at $(52,0)$. At the same time, in order to illustrate the influence of the number of IRS units on the convergence of the algorithm, we considered two cases where 


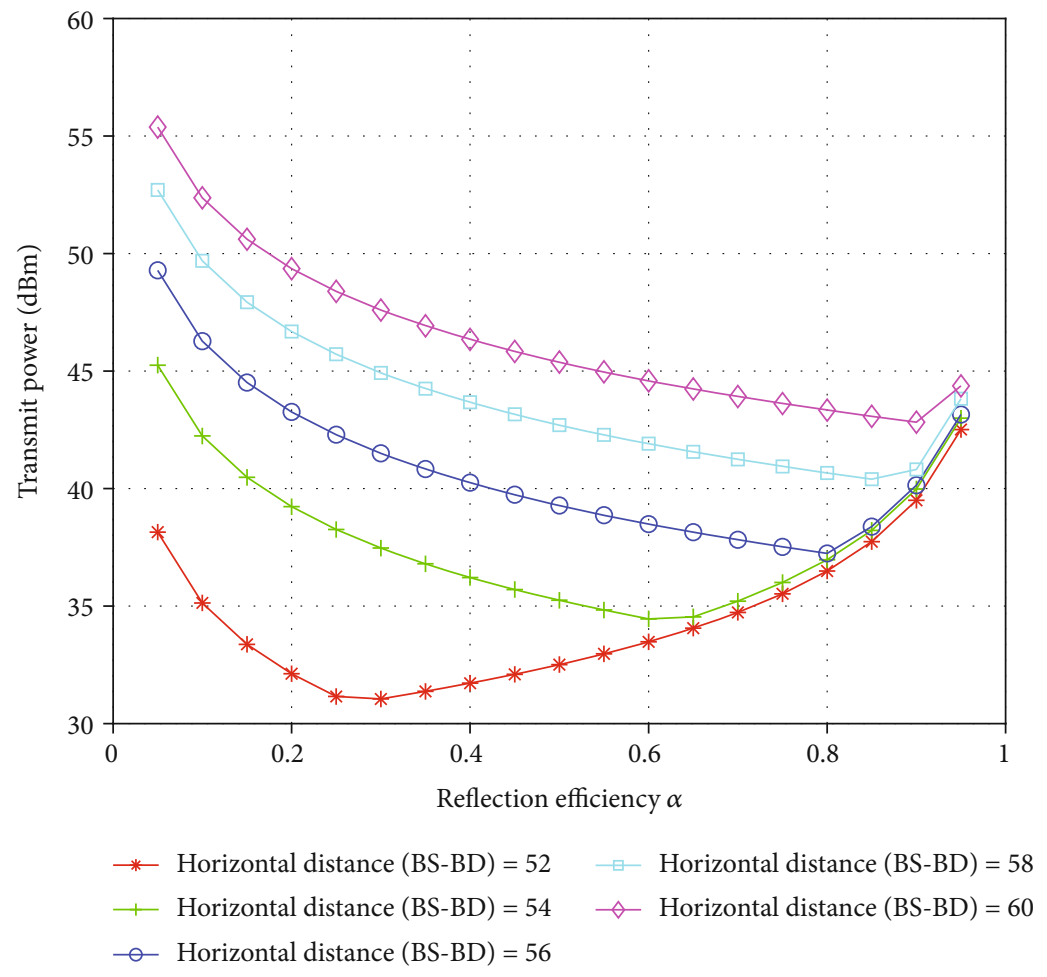

FIGURE 5: The influence of horizontal distance (BS-BD).

the number of IRS elements is $N=50$ or $N=100$. As shown in Figure 3, as the number of iterations increases, the AP's transmit power gradually decreases and tends to stabilize. This shows that the proposed algorithm has good convergence. As shown in Figure 3, when considering the impact of the threshold on the proposed algorithm, the transmit power can converge at most in three iterations. That is to say, when the maximum number of iterations is set to 3 , the proposed algorithm can obtain satisfactory transmit power. Since the complexity of the algorithm is proportional to the number of iterations, the more iterations, the lower the complexity. Therefore, Figure 3 also proves that the complexity of the proposed algorithm is low. At the same time, we can see from Figure 3 that the greater the number of IRS units, the smaller the transmit power required by the AP. And the performance of $\alpha=0.4$ is better than the performance of $\alpha$ $=0.8$.

Although Figure 3 shows the effect of different reflection efficiencies on system performance, it does not reflect the optimal reflection efficiency required by the proposed algorithm. In Figure 4, we show the effect of different reflection efficiencies on AP transmit power. We can see that although increasing the number of IRS units can reduce the transmission power, the optimal reflection coefficient setting has nothing to do with the number of reflection units. At the same time, we can see that the performance of the proposed algorithm is better than that of the system without IRS assistance.

However, it can be seen from Figure 5 that the choice of the best reflection coefficient is related to horizontal distance between AP and BD. We can see from Figure 5 that as the horizontal distance between AP and BD increases, the optimal emission coefficient increases. We can also see from Figure 5 that as the horizontal distance between AP and BD increases, the transmission power required to ensure the QoS also increases.

\section{Conclusions}

Intelligent reflector surfaces (IRSs) can improve the radio frequency environment by adjusting the phase and amplitude of the incident signal, which provides the possibility for the widespread deployment of AmBC. In this paper, we introduce a novel IRS and AmBC joint design method. This method is based on the joint design of an iterative beamforming vector, IRS phase shift, and reflection coefficient to minimize the AP's transmit power. This method can effectively reduce the transmission power of the access point, and the simulation results prove the effectiveness of this method.

\section{Data Availability}

The data in this paper is based on MATLAB simulation. According to the method described in this paper, all the data can be obtained through MATLAB.

\section{Conflicts of Interest}

The authors declare that they have no conflicts of interest. 


\section{Acknowledgments}

This work was supported by the National Key R\&D Program of China (No. 2020YFB1806700).

\section{References}

[1] Z. Zhang, Y. Xiao, Z. Ma et al., "6G wireless networks: vision, requirements, architecture, and key technologies," IEEE Vehicular Technology Magazine, vol. 14, no. 3, pp. 28-41, 2019.

[2] W. Saad, M. Bennis, and M. Chen, "A vision of $6 \mathrm{G}$ wireless systems: applications, trends, technologies, and open research problems," IEEE Network, vol. 34, no. 3, pp. 134-142, 2020.

[3] A. N. Parks, A. Liu, S. Gollakota, and J. R. Smith, "Turbocharging ambient backscatter communication," in Proceedings of the 2014 ACM Conference on SIGCOMM, pp. 619-630, Chicago, IL, USA, August 2014.

[4] K. Han and K. Huang, "Wirelessly powered backscatter communication networks: modeling, coverage, and capacity," IEEE Transactions on Wireless Communications, vol. 16, no. 4, pp. 2548-2561, 2017.

[5] W. Liu, K. Huang, X. Zhou, and S. Durrani, "Full-duplex backscatter interference networks based on time-hopping spread spectrum," IEEE Transactions on Wireless Communications, vol. 16, no. 7, pp. 4361-4377, 2017.

[6] D. T. Hoang, D. Niyato, P. Wang, D. I. Kim, and Z. Han, "Ambient backscatter: a new approach to improve network performance for RF-powered cognitive radio networks," IEEE Transactions on Communications, vol. 65, no. 9, pp. 36593674, 2017.

[7] X. Lu, D. Niyato, H. Jiang, D. I. Kim, Y. Xiao, and Z. Han, "Ambient backscatter assisted wireless powered communications," IEEE Wireless Communications, vol. 25, no. 2, pp. 170-177, 2018.

[8] N. van Huynh, D. T. Hoang, X. Lu, D. Niyato, P. Wang, and D. I. Kim, "Ambient backscatter communications: a contemporary survey," IEEE Communications Surveys \& Tutorials, vol. 20, no. 4, pp. 2889-2922, 2018.

[9] X. Kang, Y.-C. Liang, and J. Yang, "Riding on the primary: a new spectrum sharing paradigm for wireless-powered IoT devices," IEEE Transactions on Wireless Communications, vol. 17, no. 9, pp. 6335-6347, 2018.

[10] Q. Wu and R. Zhang, "Intelligent reflecting surface enhanced wireless network via joint active and passive beamforming," IEEE Transactions on Wireless Communications, vol. 18, no. 11, pp. 5394-5409, 2019.

[11] X. Jia, J. Zhao, X. Zhou, and D. Niyato, "Intelligent reflecting surface-aided backscatter communications," in GLOBECOM 2020 - 2020 IEEE Global Communications Conference, Taipei, Taiwan, December 2020.

[12] X. Yu, D. Xu, and R. Schober, "MISO wireless communication systems via intelligent reflecting surfaces," in 2019 IEEE/CIC International Conference on Communications in China (ICCC), pp. 735-740, Changchun, China, August 2019.

[13] M. M. Rana and M. M. R. Shuvo, "Localization of senor nodes using weighted least squares algorithm: comprehensive literature review and future research directions," in 2018 International Conference on Innovation in Engineering and Technology (ICIET), pp. 1-6, Dhaka, Bangladesh, December 2018.

[14] M. M. Rana, J. Kim, and W.-K. Cho, "Performance analysis of sub-carrier mapping in LTE uplink systems," in Digest of the 9th International Conference on Optical Internet (COIN 2010), pp. 1-3, Jeju, Korea (South), July 2010.

[15] S. Sun, M. Kadoch, L. Gong, and B. Rong, "Integrating network function virtualization with SDR and SDN for $4 \mathrm{G} / 5 \mathrm{G}$ networks," IEEE Network, vol. 29, no. 3, pp. 54-59, 2015.

[16] Y. Wu, B. Rong, K. Salehian, and G. Gagnon, "Cloud transmission: a new spectrum-reuse friendly digital terrestrial broadcasting transmission system," IEEE Transactions on Broadcasting, vol. 58, no. 3, pp. 329-337, 2012.

[17] B. Rong, Y. Qian, K. Lu, H.-H. Chen, and M. Guizani, "Call admission control optimization in WiMAX networks," IEEE Transactions on Vehicular Technology, vol. 57, no. 4, pp. 2509-2522, 2008.

[18] N. Chen, B. Rong, X. Zhang, and M. Kadoch, "Scalable and flexible massive MIMO precoding for 5G H-CRAN," IEEE Wireless Communications, vol. 24, no. 1, pp. 46-52, 2017.

[19] B. Rong, Y. Qian, and K. Lu, "Integrated downlink resource management for multiservice WiMAX networks," IEEE Transactions on Mobile Computing, vol. 6, no. 6, pp. 621632, 2007.

[20] S. Sun, L. Gong, B. Rong, and K. Lu, "An intelligent SDN framework for 5G heterogeneous networks," IEEE Communications Magazine, vol. 53, no. 11, pp. 142-147, 2015. 